\section{A dynamic model for solar radiation in the Tyrrhenian Basin of Central Mediterranean area}

\author{
Alessia Naccarato \\ Department of Economics, Roma Tre \\ University, Rome, Italy
}

\section{Abstract}

Studying the level of solar radiation is important for problems related to both environmental pollution and alternative energy development. In this work a space-time model for solar radiation in the Tyrrhenian basin is presented. Three main features of the model must be stressed because of their importance in modelling space-time variability of a phenomenon. The first and most important one is that relations between solar radiation in different sites are an outcome of the model's estimation procedure. With this approach spatial weights are not bound to be symmetrical and proportional to distance between locations or to be constant over time. The second one is the presence of a simultaneous effect among locations as the solar radiation in one of them is a function of what simultaneously happens in all the other ones. The third main feature of the model is represented by constrained estimation on the basis of a priori knowledge about the phenomenon that allows to cope the problem of the increased number of parameters.

\section{Introduction}

Two different kinds of interest in the study of solar radiation have been generally considered. The first one is related to environmental problems. ${ }^{1}$ The level of solar radiation is strictly connected with the presence of pollutants which, besides worsening the quality of the air we breathe, cause rarefaction of the ozone reducing the ability of the stratosphere to hold the UV-C waves, which are the most dangerous for human health. Hence, monitoring the level of solar radiation is important for checking a possible increase of this level in time due to many different phenomena that have to be kept under control. The second kind of interest is due to the fact that level of solar radiation measured at a certain time on a single site depends on the behaviour of nearby stations being a function of cloud. Streams and humidity rate cause the movement of clouds and then the intensity of solar radiation measured in a given site. For this reason we need suitably flexible models to understand and predict solar radiation which as many other phenomena has a spatial diffusion that is not dependent on the distance among sites.

Very recently a third kind of interest for dynamic solar radiation models has been added and it regards the production of electricity through photovoltaic panels. In a well known paper Chegaar and Chibani ${ }^{2}$ stated: Solar energy occupies one of the most important places among the various possible alternative energy sources. An accurate knowledge of solar radiation distribution at a particular geographical location is of vital importance for the development of many solar energy devices and for estimates of their performances.

The study of solar radiation has its natural allocation in the field of space-time data analysis., ${ }^{1,-10}$ Referring to the estimation of spatial autoregressive model an important point regards spatial weights. ${ }^{11-13}$ Anselin $^{14,15}$ and Getis $^{16}$ underline the importance of considering spatial weights strongly related to some aspects of phenomena under study, which implies that they have not necessarily to be represented by a contiguity structure. According to this point of view and because of the endogenous nature of spatial weights for solar energy diffusion, we propose to obtain them in the model's estimation procedure so that no superimposition of a fixed structure, as the one in which spatial weights are proportional to distance between locations, is made. In this way spatial weights are not bound to be symmetrical as stated in Deng ${ }^{17}$ and Cliff and $\operatorname{Ord}^{18}$ nor to be constant over time since they may change according to the lag considered. Spatial weights will then take into account the intensity and the direction of influence that a single station, in a given time, has on the whole spatial system and vice versa.

The first relevant feature of the proposed model is then the attempt to catch the spatial effect together with the temporal one, regardless of the hypothesis that a greater proximity between different places implies a higher spatial correlation. If distance between spatial locations has any effect on the diffusion of the phenomenon, such effect will be caught over by the model through the estimated spatial weights.

A second peculiar aspect of the model presented here is the simultaneous dependence of solar radiation in one location from values observed at current time in the remaining ones in order to catch the purely spatial effect; which allows to take into account the values in other locations at contemporary time $t$ to update the dependent variable at the location under exam.

The simultaneous dependence of the current value of solar energy in all locations can be seen as a second relevant features of the
Correspondence: Alessia Naccarato, Department of Economics, Roma Tre University, via Silvio D'Amico 77, 00145 Rome, Italy.

Tel. +39.06.57335651 - Fax: +39.06 .57335771 .

E-mail: alessia.naccarato@uniroma3.it

Key words: solar radiation, space-time models, simultaneous effect, constrained maximum likelihood estimator.

Conflict of interests: the author declares no potential conflict of interest.

Received for publication: 30 May 2013

Revision received: 5 February 2014.

Accepted for publication: 7 February 2014.

This work is licensed under a Creative Commons Attribution 3.0 License (by-nc 3.0).

(C) Copyright A. Naccarato, 2014

Licensee PAGEPress, Italy

Global Meteorology 2014; 3:4896

doi:10.4081/gm.2014.4896

model here presented. It must be stressed that when only lagged values at time $t-k(k=1,2$, ...) are considered a relevant loss of information takes place.

On the other hand it must be recognized that simultaneity of relations makes the estimation problem more difficult because of the increase in the number of parameters to be estimated.

In this work we propose to face it using identifying restrictions on space-time variability of the unknown parameters introducing constraints on the basis of a priori knowledge about the phenomenon. To this extent it must be stressed that the imposition of constraints on spatial weight - that have anyway to be tested - has a lesser impact on the estimation of the model than a completely arbitrary choice of their values according to prior decisions. While in a preceding paper an estimation procedure based on Two Stage Least Squares principle has been proposed, ${ }^{19}$ in this work we consider Maximum Likelihood Estimators of the whole set of parameters of the model both for constrained and unconstrained estimation. A preliminary version about some of the results here presented has been given in a previous paper. ${ }^{20}$ The model here considered expresses solar radiation at time $t$, in a given site, as a linear function of past observations in the same site, of past and simultaneous observations in the remaining ones and of the simultaneous observations of the pure exogenous effect of sunshine duration. ${ }^{21,22}$

This article is organized as follows. First, the space-time model including and not including simultaneous spatial effect is presented in order to establish notation. In the Results section, the dynamic model for spatial diffusion of 
solar radiation in the Tyrrhenian basin is presented, showing how the proposed procedure works in determining the spatio-temporal structure of the data. In the Discussion, a comparison with the model without contemporaneous effect is carried out. Few word of conclusion end the work.

\section{Materials and Methods}

Disregarding for the moment the presence of the pure exogenous variable and concentrating only on the spatial structure of the model, let us consider a spatial system in which there are $S$ places $i=1, \mathrm{~K}, \mathrm{~S}$ and $K$ temporal lags $k=1, \mathrm{~K}, K$. Let us indicate with the superscript the spatial reference and the subscript the time one, hence $y_{t}^{j}$ will be the value of solar radiation measured at time $t$ in place $j$. The linear model for each one of the $S$ station, is:

$$
\begin{aligned}
& y_{t}^{\prime}=\beta_{1}^{i} y_{t-1}^{i}+\beta_{2}^{i} y_{t-2}^{\prime}+\mathbf{L}+\beta_{K}^{i} y_{t-K}^{\prime}+
\end{aligned}
$$

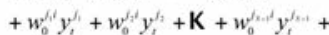

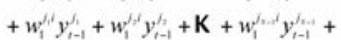

$$
\begin{aligned}
& +\mathrm{K}+
\end{aligned}
$$

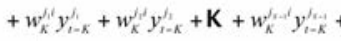

$$
\begin{aligned}
& +u^{\prime}
\end{aligned}
$$

In (eq. 1) for simplicity the hypothesis that the autoregressive component and the spacetime component have the same maximum order of lag is done; anyway, the structure of the model would be the same even in the case the lag order of the two components is not the same.

With regard to the whole spatial system the model above can be written in the following way:

$$
\begin{aligned}
& Y_{(0)}=\underset{T \times S}{Y_{(1)}} \underset{S \times S}{\beta_{1}}+\mathbf{K}+Y_{T \times S} \underset{S \times S}{\beta_{S}}+ \\
& +Y_{(0)} W_{0}+
\end{aligned}
$$

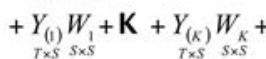

$$
\begin{aligned}
& +\underset{T \times S}{U_{(0)}}
\end{aligned}
$$

where as usual $T$ is the number of observations, $Y_{(0)}$ is the matrix of current values of variables $Y$ in the $\mathrm{S}$ stations and $Y_{(1)}, Y_{(2)}, \mathrm{K}$ are similarly defined as matrices of the lagged variable of order $1,2, \ldots ; U_{(0)}$ is the matrix of error components in the $S$ stations about which the usual hypothesis are made.

The matrix of spatial coefficients $\underset{s \times s}{W_{k}}$, with $0 \leq \mathrm{k} \leq \mathrm{K}$, is defined as follows:

$$
W_{s \times s}=\left[\begin{array}{cccccc}
0 & w_{k}^{12} & \mathbf{L} & w_{k}^{11} & \mathbf{L} & w_{k}^{1 s} \\
w_{k}^{21} & 0 & \mathbf{L} & w_{k}^{2 i} & \mathbf{L} & w_{k}^{2 s} \\
\mathbf{M} & \mathbf{M} & \mathbf{M} & \mathbf{M} & \mathbf{M} & \mathbf{M} \\
w_{k}^{11} & w_{k}^{12} & \mathbf{L} & 0 & \mathbf{L} & w_{k}^{i s} \\
\mathbf{M} & \mathbf{M} & \mathbf{M} & \mathbf{M} & \mathbf{M} & \mathbf{M} \\
w_{k}^{s 1} & w_{k}^{s 2} & \mathbf{L} & w_{k}^{s i} & \mathbf{L} & 0
\end{array}\right]
$$

where the generic coefficient $w_{k}^{i j}$ measures the effect that the phenomenon

observed in the station $i$ at time $t$ - $k$ has on the station $j$ at time $t$; while:

$$
\beta_{s \times S}=\left[\begin{array}{llllll}
\beta_{k}^{1} & 0 & \mathbf{L} & 0 & \mathbf{L} & 0 \\
0 & \beta_{k}^{2} & \mathbf{L} & 0 & \mathbf{L} & 0 \\
\mathbf{M} & \mathbf{M} & \mathbf{M} & \mathbf{M} & \mathbf{M} & \mathbf{M} \\
0 & 0 & \mathbf{L} & \beta_{k}^{i} & \mathbf{L} & 0 \\
\mathbf{M} & \mathbf{M} & \mathbf{M} & \mathbf{M} & \mathbf{M} & \mathbf{M} \\
0 & 0 & \mathbf{L} & 0 & \mathbf{L} & \beta_{k}^{s}
\end{array}\right]
$$

is the matrix of the coefficients of the autoregressive variables, where, indicates the effect that the phenomenon recorded in the $i$-th station at time $t-k$ has on $Y$ observed in the same station but at time $t$.

Eq. 2 can also be written as:

$$
\begin{aligned}
& Y_{(0)}^{T}=W_{0}^{T} Y_{(0)}^{T}+\left(W_{1}^{T}+\beta_{1}^{T}\right) Y_{(0)}^{T}+K+\left(W_{\kappa}^{T}+\beta_{\kappa}^{T}\right) Y_{(k)}^{T}+U_{(0)}^{T} \text { (eq. 3) } \\
& \text { or } \\
& \left(I_{s}-W_{+}^{T}\right)_{(0)}^{\tau}-\left(W_{1}^{T}+\beta_{i}^{T}\right)_{(0)}^{T}+\mathrm{K}+\left(W_{\kappa}^{T}+\beta_{\kappa}^{\tau}\right)_{(k)}^{T}+U_{(0)}^{T} \text { (eq. 4) }
\end{aligned}
$$

and defining

$$
\begin{gathered}
\left(I_{S}-W_{0}^{T}\right)=A_{0} \\
\left(W_{\star}^{T}+\beta_{\star}^{\tau}\right)=A_{\star}, 1 \leq k \leq K
\end{gathered}
$$

eq. 4 becomes:

$$
A_{0} Y_{(0)}^{T}=A_{1} Y_{(0)}^{T}+\mathbf{K}+A_{K} Y_{(K)}^{T}+U_{(0)}^{T}
$$

We will refer to eq. 6 as the STSE (K) model. To better understand the performance of model STSE (2), we have estimated an ST (2) model of the form

$$
Y_{(0)}^{T}=A_{1} Y_{(0)}^{T}+\mathbf{K}+A_{K} Y_{(K)}^{T}+U_{(0)}^{T}
$$

so that a comparison can be made between the two models. In both cases the effect of the purely exogenous variable sunshine duration has been introduced.

As it is well known when the model includes the simultaneous spatial component ordinary least square leads to inconsistent estimators of unknown parameters because of correlation between contemporaneous observed errors in

different locations, $E\left(u_{t}^{i} u_{t}^{j}\right) \neq 0$. Therefore, under the hypothesis that the process is normally distributed, maximum likelihood estimation have been considered to obtain consistent estimators. In the search of a parsimonious model which allows not only to estimate spatial weights but also to find the structure of spatial influence and the underlining effect of contiguity a maximum likelihood constrained estimation procedure has been developed. To test the significance of constraint on variables' coefficients likelihood ratio (LR) test has been performed. Since for both constrained and unconstrained model the maximization of loglikelihood function requires the use of an iterative algorithm, the test statistics will be approximated. In case large samples are available - as it is in the present study - the approximation can be neglected.

The data used in our application are the spatio-temporal series of daily average of solar radiation and daily sunshine duration concerning the fifteen Italian meteorological stations of the Tyrrhenian Basin - Pisa San Giusto (01), Elba (02), Pianosa (03), Vigna di Valle (04), Roma Ciampino (05), Ponza (06), Napoli Capo di Chino (07), Capo Palinuro (08), Messina (09), Ustica (10), Trapani Birgi (11), Pantelleria (12), Cagliari Elmas (13), Capo Bellavista (14), Olbia Costa Smeralda (15) during the period January $1^{\text {st }} 1991$ to $31^{\text {st }}$ December 1998, provided by the Italian Air Force Meteorological Station of Vigna di Valle (Rome).

With more details, the solar radiation series reports the daily average solar radiation levels measured at ground level using a pyrheliometer; the unit of measurement was mega-joules per square meter $(\mathrm{MJ} / \mathrm{m} 2)$. The sunshine duration refers to the duration (in hours and tenths of hours) of sunlight when the sun is situated at a height of at least $3^{\circ}$ above the horizon. A heliophonograph was used to measure sunshine duration.

\section{Results}

The model STSE(2) has the following form:

$$
y_{t}=\alpha+C_{0} x_{t}+A_{0} y_{t}+\sum_{k=1}^{2} A_{k} y_{t-k}+u_{t}
$$

where in addition to the presence of the simultaneous and space-time effect we introduce sunshine duration as the unique truly exogenous variable in the model; its coefficients matrix is indicated with $\mathrm{C}_{0}$. The order of lag in STSE model has been fixed at 2 , since for all the series considered the estimated global autocorrelation function goes to zero very slowly and in a straight way.

To cope with the main problem in the use of the proposed model, i.e. the high number of parameters to be estimated, we introduce con- 
straint based on available $a$ priori information about meteorological characteristics of the climatic area considered. The fifteen sites considered are under the continuous and predominant influence of the streams coming from the English Channel and the Gulf of Gascony, which cause the movements of clouds in the same directions for two or three days; hence we assumed the spatio-temporal effect of the phenomenon to be constant in the same period. This ends up to suppose that the extradiagonal elements of the matrix $A_{2}$ should be the same as those of the matrix $A_{1}$. To verify this hypothesis we used the LR test to compare the fit of two models nested one in the other. ${ }^{23}$ The test is based on the ratio between the maximum of constrained and unconstrained likelihood function, in which the equality of extra-diagonal elements of the two matrices is imposed element by element. We then introduced in the estimation procedure the constraints of constancy only for those coefficients for which the equality was not rejected by LR test. LR test shows also that the same kind of constraints do not hold for simultaneous effect coefficients in $A_{0}$.

In Tables 1, 2 and 3 we show the estimated matrices of coefficients obtained maximizing the log-likelihood function under constraints resulting from LR test. In matrix $A_{2}$ (Table 3) we reported in italics the parameters which remain constant between lag 1 and lag 2 . In this way the number of parameters to be estimated is reduced and the fitting of the data shows up to be quite good. To see this we make use of squared multiple correlation coefficient $\left(R^{2}\right)$ whose range is from zero to one: values closer to one indicate a good fitting of the esti- mated model. ${ }^{24}$ In the present case the value of $R^{2}$ is equal to 0.85 which is close enough to one to mean a quite good fitting.

In Figures 1 and 2 influences of each station on the others (i.e. the estimated elements of matrices $A_{0}, A_{1}$, and $A_{2}$, that from now on we will simply call coefficients) are represented by means of arrows: white arrows for the simultaneous effect, black ones for the effect at lag1 and grey for those at lag2. Arrows' greater thickness indicates stronger space and spacetime effects. In the graphic representation we have decided to show only cases in which the estimated coefficients are grater than 0.6 to better represent the main structure of climatic influences. In Figure 1 white arrows (simultaneous influence of each station on the others) show the presence of roughly defined micro areas such as the one of Tuscany (stations 1,2,

Table 1. Estimated coefficient for matrix $A_{0}$ in STSE(2) model.

\begin{tabular}{|c|c|c|c|c|c|c|c|c|c|c|c|c|c|c|c|}
\hline $\mathrm{A}_{0}$ & 01 & 02 & 03 & 04 & 05 & 06 & 07 & 08 & 09 & 10 & 11 & 12 & 13 & 14 & 15 \\
\hline 01 & -1 & 0.54 & 0.82 & 0.59 & 0.69 & 0.23 & & & & & & & & & 0.33 \\
\hline 02 & 0.74 & -1 & 0.81 & 0.48 & 0.71 & 0.56 & 0.31 & 0.46 & & 2 & & & & 0.18 & 0.64 \\
\hline 03 & 0.52 & 0.63 & -1 & 0.14 & 0.33 & 0.49 & 0.28 & 0.14 & & & & & & 0.39 & 0.53 \\
\hline 04 & 0.84 & 0.79 & 0.72 & -1 & 0.61 & 0.54 & 0.70 & & & 0.15 & & & & 0.44 & 0.41 \\
\hline 05 & 0.33 & 0.25 & 0.49 & 0.65 & -1 & 0.69 & 0.74 & 0.21 & 0.41 & 0.12 & & & & 0.38 & 0.12 \\
\hline 06 & & & & 0.18 & 0.42 & -1 & 0.53 & 0.86 & 0.74 & 0.59 & & & & & \\
\hline 07 & & & & & & 0.89 & -1 & 0.81 & 0.52 & 0.62 & & & & & \\
\hline 08 & & & & & & & & -1 & 0.79 & & & & & & \\
\hline 09 & & & & & & & & 0.83 & -1 & & 0.73 & & & & \\
\hline 10 & & & & & & & 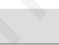 & & & -1 & 0.64 & 0.58 & & & \\
\hline 11 & & & & & & & & & & 0.75 & -1 & 0.59 & & & \\
\hline 12 & & & & & & 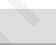 & & & & & 0.48 & -1 & & & \\
\hline 13 & & & & & & & & & & & 0.71 & 0.75 & -1 & & \\
\hline 14 & & & & & 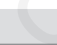 & & 0.11 & 0.46 & 0.42 & 0.54 & 0.58 & 0.22 & & -1 & \\
\hline 15 & & & & 0.25 & 0.36 & 0.78 & 0.65 & & & 0.69 & 0.52 & & & & -1 \\
\hline
\end{tabular}

Table 2. Estimated coefficient for matrix $A_{1}$ in $\operatorname{STSE}(2)$ model.

\begin{tabular}{|c|c|c|c|c|c|c|c|c|c|c|c|c|c|c|c|}
\hline $\mathbf{A}_{1}$ & 01 & 02 & 03 & 04 & 05 & 06 & 07 & 08 & 09 & 10 & 11 & 12 & 13 & 14 & 15 \\
\hline 01 & 0.28 & 0.12 & 0.31 & 0.57 & 0.67 & 0.11 & 0.25 & & & & & & & & \\
\hline 02 & 0.18 & 0.31 & & 0.32 & 0.43 & 0.38 & 0.19 & 0.11 & & & & & & & 0.28 \\
\hline 03 & 0.23 & 0.26 & 0.44 & & & 0.16 & 0.12 & 0.09 & 0.10 & & & & & 0.13 & \\
\hline 04 & & & & 0.13 & & 0.23 & 0.61 & 0.52 & -0.32 & 0.27 & 0.09 & & & & \\
\hline 05 & & & & & 0.81 & 0.29 & 0.66 & 0.56 & 0.29 & 0.31 & 0.11 & & & & \\
\hline 06 & & & & & & 0.63 & 0.33 & 0.73 & 0.41 & 0.64 & 0.37 & & & & \\
\hline 07 & & & & & & & 0.29 & 0.59 & 0.61 & 0.72 & 0.62 & & & & \\
\hline 08 & & & & & & & & 0.52 & 0.34 & & 0.19 & & & & \\
\hline 09 & & & & & & & & & 0.65 & & 0.23 & & & & \\
\hline 10 & & & & & & & & & & 0.32 & 0.47 & 0.36 & & & \\
\hline 11 & & & & & & & & & & & 0.15 & 0.48 & & & \\
\hline 12 & & & & & & & & & & & 0.41 & 0.18 & & & \\
\hline 13 & & & & & & & & 0.13 & 0.11 & 0.55 & 0.68 & 0.64 & 0.27 & & \\
\hline 14 & & & & & & 0.58 & & 0.22 & 0.25 & 0.79 & -0.27 & 0.37 & 0.29 & 0.23 & \\
\hline 15 & & & & 0.31 & 0.33 & -0.24 & -0.15 & & & 0.28 & 0.23 & & & 0.68 & 0.73 \\
\hline
\end{tabular}


$3,4,5)$, the area from Naples to Sicily (stations $6,7,8,9)$. On all the islands and on western Sicily (stations $6,10,11,12$ ) is strongly evident the almost one directional influence of the Sardinian ones (stations 13, 14, 15). It must be noticed that white arrows show up a higher inter connection of sites than those shown by black and grey ones. It has also to be noticed that at lag1 and 2 (black and grey arrows) there are spatial effect among farther sites.

In many cases while contemporaneous effect coefficients are different from zero, they are zero at lag one and lag two; vice versa coefficients which are different from zero at the two lags, are zero among the contemporaneous ones. Stations 2 (Elba) and 3 (Pianosa) show, for instance, a reciprocal contemporaneous effect which is not present at lag one or at lag two. Vice versa station 4 (Vigna di Valle) has an influence on station 8 (Capo Palinuro) only at lags one and two. Simultaneous effect is generally detected between closer sites while for more distant ones prevail the effect at lag one and two. In accordance with the northsouth main direction of clouds' streams. With regard to the pure autoregressive coefficients (diagonal elements of $A_{1}$ and $A_{2}$ in Tables 2 and 3 ) it is worthwhile to notice that a generalized decrease of coefficients passing from lag one to lag two is shown.

\section{Discussion}

In order to evaluate the importance of simultaneous effects, we compare the results obtained with STES(2) with those obtained with model ST(2) which is without it:

$$
y_{t}=\alpha+C_{0} x_{t}+\sum_{k=1}^{2} A_{k} y_{t-k}+u_{t}
$$

First of all it has to be noticed that in the estimation procedure a non stationarity in mean has been detected that was not present in the preceding model. To eliminate it first order differentiation has been considered. The differentiated series show an autoregressive scheme of second order for all the sites. Spatio-temporal effects for lags greater than two were also taken into account but their inclusion in the models was not significant. It has to be noticed that also in this case the model fit well the data $\left(R^{2}=0.77\right)$.

As far as the comparison between the two models is concerned, the number of coefficient considered in Figures 1 and 2 related to black arrows (first lag) is almost the same but in ST(2) model the values of the coefficients are generally greater than those in STSE(2). Hence, it seems that the omission of the variable related to the pure spatial (i.e. contemporaneous) effect has the increase of coefficients at lag1 as a consequence.

It can be also noticed that the number of relations considered for lag2 (grey arrows) is greater in STSE(2) than in ST(2), as if the presence of $A_{0}$ makes the model more effective.

Finally, we want to stress that the improvement in the value of $R^{2}$ obtained in STSE(2) with respect to ST(2) (from 0.77 to 0.85 ) is in our opinion sufficiently strong to confirm that the presence of a simultaneous effect is essen- tial for a better representation of solar radiation diffusion.

\section{Conclusions}

In this work we present a dynamic model for solar radiation that is very flexible and appropriate to understand the directional effects of non symmetric variability both over space and time. In this model we consider the presence of a simultaneous effect of spatial locations on each other. The model defines a non symmetric relationship both for contemporaneous and for lagged components.

As far as the presence of pure spatial weights is concerned, it is worthwhile to stress the better performance of the model with contemporaneous non symmetric effect with respect to the one in which simultaneity is not taken into account. The introduction of contemporary effect improves substantially the performance of the model.

To assume non-symmetry of relations between sites at every lag, however, involves a larger number of parameters to be estimated even if it has to be stressed that this difficulty can be irrelevant if the number of available observations is large. However, even in that case constraints on the unknown coefficients can be formulated on the basis of a priori information about the spatial and spatio-temporal diffusion of the process. In order to face this problem we used a priori information about the movements of the clouds on the target area. The estimated spatio-temporal coefficients of the model are in accordance with the

Table 3. Estimated coefficient for matrix $A_{2}$ in STSE(2) model.

\begin{tabular}{|c|c|c|c|c|c|c|c|c|c|c|c|c|c|c|c|}
\hline $\mathbf{A}_{2}$ & 01 & 02 & 03 & 04 & 05 & 06 & 07 & 08 & 09 & 10 & 11 & 12 & 13 & 14 & 15 \\
\hline 01 & 0.09 & & & 0.57 & 0.67 & 0.09 & 0.14 & & & & & & & & \\
\hline 02 & & 0.14 & & 0.39 & 0.43 & 0.38 & 0.19 & 0.11 & & & & & & & \\
\hline 03 & 0.23 & 0.26 & 0.31 & & & & 0.02 & 0.12 & 0.09 & 0.10 & & & & & \\
\hline 04 & & & & 0.02 & & 0.23 & 0.61 & 0.52 & -0.32 & 0.27 & 0.05 & 0.02 & & & \\
\hline 05 & & & & & 0.12 & 0.29 & 0.66 & 0.56 & 0.29 & 0.31 & 0.07 & 0.06 & & & \\
\hline 06 & & & & & 0.08 & 041 & 0.33 & 0.73 & 0.41 & 0.32 & & & & & \\
\hline 07 & & & & & & & 0.07 & 0.59 & 0.61 & 0.51 & 0.58 & & & & \\
\hline 08 & & & & & & & & 0.11 & 0.34 & 0.06 & 0.19 & & & & \\
\hline 09 & & & & & & & & & 0.35 & 0.05 & 0.23 & & & & \\
\hline 10 & & & & & & & & & & 0.18 & 0.47 & 0.36 & & & \\
\hline 11 & & & & & & & & & & 0.01 & 0.06 & 0.48 & & & \\
\hline 12 & & & & & & & & & & & 0.01 & 0.21 & & & \\
\hline 13 & & & & & & & & & & 0.55 & 0.68 & 0.64 & 0.14 & & \\
\hline 14 & & & & & & 0.58 & & 0.22 & 0.25 & 0.63 & 0.25 & 0.37 & 0.29 & 0.08 & \\
\hline 15 & & & & 0.31 & 0.33 & 0.09 & & & & 0.28 & 0.23 & & & 0.47 & 0.52 \\
\hline
\end{tabular}




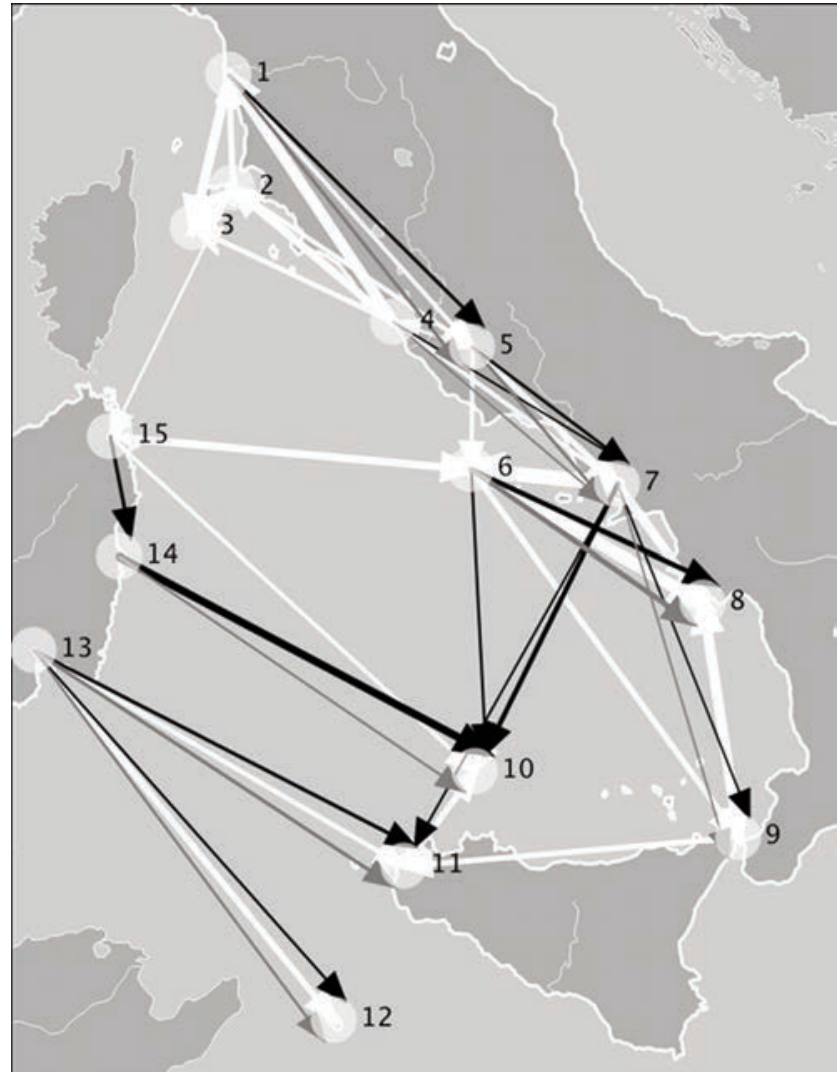

Figure 1. Main spatial effects (coefficient>0.6) for STSE(2) model. White arrows represent current effects, black arrows stand for lag1 effects, while grey arrows show lag2 effects. Meteorological stations in Tyrrhenian Basin are: 1=Pisa San Giusto; 2=Elba; 3=Pianosa; 4=Vigna di Valle; 5=Roma Ciampino; 6=Ponza; 7=Napoli Capo di Chino; 8=Capo Palinuro; 9=Messina; 10=Ustica; 11=Trapani Birgi; 12=Pantelleria; 13=Cagliari Elmas; 14=Capo Bellavista; 15=Olbia Costa Smeralda.

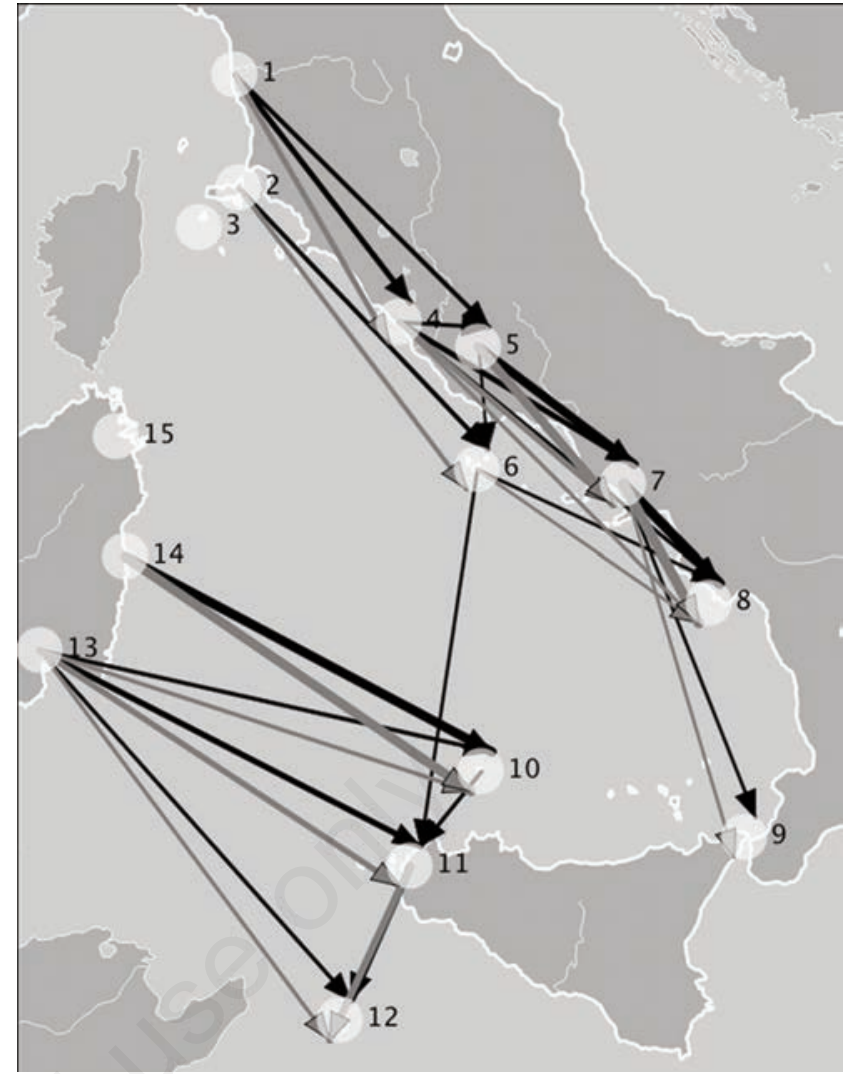

Figure 2. Main spatial effects (coefficient>0.6) for ST(2) model. Black arrows represent lag1 effects, while grey arrows stand for lag2 effects. Meteorological stations in Tyrrhenian Basin are: 1 =Pisa San Giusto; 2=Elba; 3=Pianosa; 4=Vigna di Valle; 5=Roma Ciampino; 6=Ponza; 7=Napoli Capo di Chino; 8=Capo Palinuro; 9=Messina; 10=Ustica; 11=Trapani Birgi; 12=Pantelleria; 13=Cagliari Elmas; 14=Capo Bellavista; 15=Olbia Costa Smeralda. main movements of clouds in the Mediterranean Basin. In particular they capture the effects of the main movements of clouds in the NW-SE direction as those from Tuscany Archipelago and Sardinia to the South of Italy. This fact is evident looking at the matrices of spatio-temporal coefficients where, after ordering the meteorological sites from the North to the South, we can see that almost all the under-diagonal elements are zero.

Even more clear is the representation of main spatial effects (coefficients $>0.6$ ) in Figures 1 and 2. Furthermore, the proposed model points out that spatial effects may change as a function of the temporal lag considered. As noted before, it has not been possible to hold the constancy constraints for the relations among all the sites. In particular, using simultaneous effects we can see that at lag 2 there are spatial effects among further sites.
In our opinion, while such a procedure should require the support of a priori information, it would always be appropriate to obtain a more parsimonious fit of the model. In this sense it is possible to think of a procedure that gets the definition of a parsimonious set of parameters through testing.

\section{References}

1. Evrendilek F, Ertekin C. Assessing solar radiation models using multiple variables over Turkey. Clim Dynam 2008;31:131-49.

2. Cheegar M, Chibani A. A simple method for computing solar radiation. Rev Energ Ren Chemss 2000;2000:111-5.

3. Gelfand AE, Ghosh SK, Knight JR, Sirmans CF. Spatio-temporal modeling of residential sales data. J Bus Econ Stat 1998; 16:312-21.
4. Kyriakidis PC, Journel AG. Geostatistical space-time models. Math Geol 1999;31: 651-84.

5. Cressie N, Huang HC. Classes of nonseparable spatio-temporal stationary covariance functions. J Am Stat Assoc 1999; 448:1330-40.

6. Huang HC, Cressie N. Spatio-temporal prediction of snow water equivalent using the kalman filter. Comput Stat Data An 1996;22:159-75.

7. Gneiting T. Nonseparable stationary covariance functions for space-time data. $\mathrm{J}$ Am Stat Assoc 2002;458:590-600.

8. Lee H, Ghosh SK. A reparametrization approach for dynamic space-time models. J Stat Theory Pract 2008;2:1-14.

9. Glasbey CA, Allcroft DJ. A spatiotemporal auto-regressive moving average model for solar radiation. J Roy Stat Soc C-App) 2008;57:343-55.

10. Heuvelink GBM, Griffith DA. Space-time 
geostatistics for geography: a case study of radiation monitoring across parts of Germany. Geogr Anal 2010;42:161-79.

11. Banerjee S, Gelfand AE, Gamerman D. Spatial process modelling for univariate and multivariate dynamic spatial data. Environmetrics 2005;16:1-15.

12. Stroud JR, Muller P, Sansò B. Dynamic models for spatiotemporal data. J Roy Stat Soc B 2001;63:673-89.

13. Haining RP, Kerry R, Oliver MA. Geography, spatial data analysis, and geostatistics: an overview. Geogr Anal 2010;42:7-31.

14. Anselin L. Local indicators of spatial association: LISA. Geogr Anal 1995;27:93-115.

15. Anselin L. Spatial externalities, spatial multipliers, and spatial econometrics. Int Regional Sci Rev 2003;26:153-66.
16. Getis A. Spatial weights matrices. Geogr Anal 2009;41:404-10.

17. Deng M. An anisotropic model for spatial processes. Geogr Anal 2008;40:26-51.

18. Cliff $\mathrm{AD}$, Ord JK. What were we thinking? Geogr Anal 2009;41:351-63.

19. Naccarato A. I minimi quadrati a due stadi per la stima di serie spazio-temporali con effetto simultaneo. Quaderni di Statistica 2004;6:49-69.

20. Naccarato A, Lamberti A. VAR models for spatio-temporal structures: an application to environmental data. In: Vichi M, Monari P, Mignani S, Montanari A, eds. New developments in classification and data analysis: Proceedings of the Meeting of the Classification and Data Analysis Group of the Italian Statistical Society, Bologna, Italy. Berlin: Springer; 2003. pp 243-250.
21. Simmons PA. A mathematical representation of the incident solar energy as a function of latitude and time. Clim Dynam 1998;3:41-4.

22. Kutzbach JE, Chen G, Cheng H, et al. Potential role of winter rainfall in explaining increased moisture in the Mediterranean and Middle East during periods of maximum orbitally forced insolation seasonality. Clim Dynam 2013;42:1079-95.

23. Casella G, Berger RL. Statistical inference. 2th ed. Pacific Grove, CA, USA: Cengage Learning; 2002.

24. Chatterjee $\mathrm{S}$, Hadi AS. Sensitivity analysis in linear regression. New York, NY, USA: John Wiley \& Sons Inc.; 1988. 